\title{
The Balloon-borne Large-Aperture Submillimeter Telescope for polarization: BLAST-pol
}

G. Marsden, P. A. R. Ade, S. Benton, J. J. Bock, E. L. Chapin, et al.

G. Marsden, P. A. R. Ade, S. Benton, J. J. Bock, E. L. Chapin, J. Chung, M. J. Devlin, S. Dicker, L. Fissel, M. Griffin, J. O. Gundersen, M. Halpern, P. C. Hargrave, D. H. Hughes, J. Klein, A. Korotkov, C. J. MacTavish, P. G. Martin, T. G. Martin, T. G. Matthews, P. Mauskopf, L. Moncelsi, C. B. Netterfield, G. Novak, E. Pascale, L. Olmi, G. Patanchon, M. Rex, G. Savini, D. Scott, C. Semisch, N. Thomas, M. D. P. Truch, C. Tucker, G. S. Tucker, M. P. Viero, D. Ward-Thompson, D. V. Wiebe, "The Balloon-borne Large-Aperture Submillimeter Telescope for polarization: BLAST-pol," Proc. SPIE 7020, Millimeter and Submillimeter Detectors and Instrumentation for Astronomy IV, 702002 (18 July 2008); doi: 10.1117/12.788413

SPIE Event: SPIE Astronomical Telescopes + Instrumentation, 2008, Marseille, France 


\title{
The Balloon-borne Large-Aperture Submillimeter Telescope for polarization: BLAST-pol
}

G. Marsden ${ }^{a}$ P. A. R. Ade, ${ }^{b}$ S. Benton, ${ }^{c}$ J. J. Bock,${ }^{d, e}$ E. L. Chapin, ${ }^{a}$ J. Chung, ${ }^{a, c}$ M. J. Devlin, ${ }^{f}$ S. Dicker,${ }^{f}$ L. Fissel,${ }^{c}$ M. Griffin, ${ }^{b}$ J. O. Gundersen, ${ }^{g}$ M. Halpern,${ }^{a}$ P. C. Hargrave, ${ }^{b}$ D. H. Hughes,${ }^{h}$ J. Klein, ${ }^{f}$ A. Korotkov ${ }^{i}$ C. J. MacTavish, ${ }^{c}$ P. G. Martin,${ }^{j, k}$ T. G. Martin,${ }^{c}$ T. G. Matthews, ${ }^{l}$ P. Mauskopf,${ }^{b}$ L. Moncelsi, ${ }^{b}$ C. B. Netterfield, ${ }^{c, k}$ G. Novak, ${ }^{l}$ E. Pascale, ${ }^{b}$ L. Olmi,${ }^{m, n}$ G. Patanchon, ${ }^{a, o}$ M. Rex,${ }^{f}$ G. Savini,${ }^{b}$ D. Scott,${ }^{a}$ C. Semisch,${ }^{f}$ N. Thomas,${ }^{g}$ M. D. P. Truch, ${ }^{f}$ C. Tucker,${ }^{b}$ G. S. Tucker,${ }^{i}$ M. P. Viero, ${ }^{k}$ D. Ward-Thompson,${ }^{b}$ D. V. Wiebe ${ }^{c}$

${ }^{a}$ Department of Physics \& Astronomy, University of British Columbia, 6224 Agricultural Road, Vancouver, BC V6T 1Z1, Canada;

${ }^{b}$ Department of Physics \& Astronomy, Cardiff University, 5 The Parade, Cardiff, CF24 3AA, $\mathrm{UK}$;

${ }^{c}$ Department of Physics, University of Toronto, 60 St. George Street, Toronto, ON M5S 1A7, Canada;

${ }^{d}$ Jet Propulsion Laboratory, Pasadena, CA 91109-8099;

${ }^{e}$ Observational Cosmology, MS 59-33, California Institute of Technology, Pasadena, CA 91125;

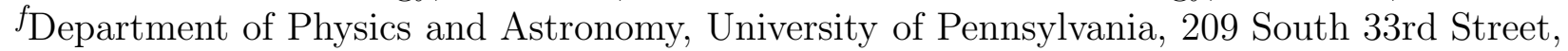
Philadelphia, PA 19104;

${ }^{g}$ Department of Physics, University of Miami, 1320 Campo Sano Drive, Coral Gables, FL 33146 ;

${ }^{h}$ Instituto Nacional de Astrofísica Óptica y Electrónica (INAOE), Aptdo. Postal 51 y 72000 Puebla, Mexico;

${ }^{i}$ Department of Physics, Brown University, 182 Hope Street, Providence, RI 02912;

${ }^{j}$ Canadian Institute for Theoretical Astrophysics, University of Toronto, 60 St. George Street, Toronto, ON M5S 3H8, Canada;

${ }^{k}$ Department of Astronomy \& Astrophysics, University of Toronto, 50 St. George Street, Toronto, ON M5S 3H4, Canada;

${ }^{l}$ Department of Physics and Astronomy, Northwestern University, 2145 Sheridan Road, Evanston, IL 60208-3112;

${ }^{m}$ Istituto di Radioastronomia, Largo E. Fermi 5, I-50125, Firenze, Italy; ${ }^{n}$ University of Puerto Rico, Rio Piedras Campus, Physics Dept., Box 23343, UPR station, San Juan, Puerto Rico;

${ }^{\circ}$ Laboratoire APC, 10, rue Alice Domon et Léonie Duquet 75205 Paris, France;

\begin{abstract}
The Balloon-borne Large Aperture Submillimeter Telescope (BLAST) is a sub-orbital experiment designed to study the process of star formation in local galaxies (including the Milky Way) and in galaxies at cosmological distances. Using a $2 \mathrm{~m}$ Cassegrain telescope, BLAST images the sky onto a focal plane, which consists of 270 bolometric detectors split between three arrays, observing simultaneously in $30 \%$ wide bands, centered at 250 , 350 , and $500 \mu \mathrm{m}$. The diffraction-limited optical system provides a resolution of $30^{\prime \prime}$ at $250 \mu \mathrm{m}$. The pointing
\end{abstract}

Further author information: (Send correspondence to G. Marsden)

G. Marsden: E-mail: gmarsden@phas.ubc.ca, Telephone: 604-822-6709

Millimeter and Submillimeter Detectors and Instrumentation for Astronomy IV edited by William D. Duncan, Wayne S. Holland, Stafford Withington, Jonas Zmuidzinas Proc. of SPIE Vol. 7020, 702002, (2008) -0277-786X/08/\$18 - doi: 10.1117/12.788413 
system enables raster-like scans with a positional accuracy of $\sim 30^{\prime \prime}$, reconstructed to better than $5^{\prime \prime}$ rms in postflight analysis. BLAST had two successful flights, from the Arctic in 2005, and from Antarctica in 2006, which provided the first high-resolution and large-area $\left(\sim 0.8-200 \mathrm{deg}^{2}\right)$ submillimeter surveys at these wavelengths. As a pathfinder for the SPIRE instrument on Herschel, BLAST shares with the ESA satellite similar focal plane technology and scientific motivation. A third flight in 2009 will see the instrument modified to be polarizationsensitive (BLAST-pol). With its unprecedented mapping speed and resolution, BLAST-pol will provide insights into Galactic star-forming nurseries, and give the necessary link between the larger, coarse resolution surveys and the narrow, resolved observations of star-forming structures from space and ground based instruments being commissioned in the next 5 years.

Keywords: submillimeter — stars:formation — instrumentation:miscellaneous — balloons — polarization

\section{INTRODUCTION}

The Balloon-borne Large Aperture Submillimeter Telescope (BLAST) is a stratospheric $2 \mathrm{~m}$ telescope which observes the sky with bolometric detectors operating in three $30 \%$ wide bands at 250,350 , and $500 \mu \mathrm{m}$. The diffraction-limited optics are designed to provide BLAST with a resolution of $30^{\prime \prime}, 42^{\prime \prime}$, and $60^{\prime \prime}$ at the three wavebands, respectively. The detectors and cold optics are adapted from those to be used on the SPIRE instrument on Herschel. ${ }^{1}$

BLAST addresses important Galactic and cosmological questions regarding the formation and evolution of stars, galaxies and clusters ${ }^{2}$ by providing the first large-area $\left(0.8-200 \mathrm{deg}^{2}\right)$ surveys of unique spectral-coverage, angular resolution and sensitivity. The primary scientific goals of BLAST are: (1) to conduct confusion-limited and shallower wide-area extragalactic surveys to constrain the redshift-distribution, star formation history, and evolution of optically-obscured luminous galaxies by measuring photometric redshifts (derived from the BLAST colors and complementary data ${ }^{3}$ ); (2) to study the spatial clustering of this population; (3) to improve our understanding of the earliest stages of star formation by determining the physical properties and mass-function of cold pre-stellar cores and the efficiency of star formation within different Galactic environments; and (4) to investigate the nature and structure of the interstellar medium by making the highest resolution maps to date of diffuse Galactic emission at these wavelengths.

BLAST has had two Long Duration Balloon (LDB) flights. The first was a 4-day flight from Kiruna, Sweden in June of 2005 (BLAST05). The primary mirror was slightly damaged on launch or ascent preventing sensitive extragalactic observations. However, we were able to observe multiple Galactic targets resulting in a successful series of observations. ${ }^{4-7}$ The instrument was quickly repaired and flew again from Antarctica in December 2006 with an 11-day flight (BLAST06). This time the entire instrument worked perfectly. We obtained multiple deep large-area Galactic and extragalactic maps.

The termination of the flight in Antarctica resulted in the payload being dragged $200 \mathrm{~km}$ over the course of $24 \mathrm{hr}$. Fortunately, the hard drives containing the flight data were recovered. We were also able to recover the detectors, the only irreplaceable component, as well as the receiver and the primary and secondary mirrors. This allowed us to continue with our long-term plans for the program. The reconstruction of the instrument is well-underway and it is expected to be completed by mid 2009 .

The simple transformation of BLAST into BLAST-pol will make it a uniquely sensitive experiment for mapping polarized emission from Galactic dust. BLAST-pol will be a powerful instrument for studying the hotly debated question of whether it is magnetic fields or turbulence that controls star formation. In particular, we will be able to map large-scale fields over an entire Giant Molecular Cloud (GMC) with enough angular resolution to trace the fields into cloud cores and dense filaments. Scales accessible to BLAST-pol lie in between those soon accessible to Planck ( $5^{\prime}$ resolution), SCUBA-2 ( $7^{\prime \prime}$ at $\left.450 \mu \mathrm{m}\right)$ and ALMA (sub-arcsecond).

\section{SCIENCE GOALS}

\subsection{Results}

BLAST was designed to conduct confusion-limited and wide-area extragalactic and Galactic surveys at submillimeter (submm) wavelengths from an LDB platform. BLAST's wavelengths are impossible or very difficult to 
observe from even the best ground-based telescope sites. In the Kiruna flight we were able to observe multiple Galactic targets resulting in a successful series of observations (Truch et al. 2008; Pascale et al. 2008; Patanchon et al. 2007; Chapin et al. 2007; see also Fig. 1). The first results from BLAST06 are in preparation. At least 10 more publications are planned before the dataset will be released to the community. These results, important on their own, will also guide the SPIRE team in planning observations and making the best use of this mission. The team is now in the process to add polarization capability to the existing focal plane: the BLAST-pol experiment.

\subsection{Mapping the Galactic Large-Scale Magnetic Fields}

Despite many advances, fundamental questions about star formation remain unanswered. ${ }^{8}$ Is star formation regulated by magnetic fields or by turbulence? Do molecular clouds and their substructures (cores, filaments, and clumps) have lifetimes exceeding their turbulent crossing times? Magnetic fields in molecular clouds are notoriously difficult to observe. ${ }^{9,10}$ A promising method for probing these fields is far-IR/submm polarimetry. ${ }^{11,12}$ BLAST-pol will be the first submm polarimeter having both (a) sufficient mapping speed to trace fields across entire clouds, for a statistically significant sample of GMCs, and (b) sufficient spatial resolution to follow the cloud fields into the dense cores. BLAST-pol will map polarized emission from $\mathrm{Av}=100 \mathrm{mag}$ out to $\mathrm{Av}=$ 4 mag, yielding $\sim 1000$ polarization detections per cloud, for scores of clouds. No other instrument will have this capability. These data will allow the first detailed comparisons between observed GMC field maps and synthetic GMC field maps - the latter derived from numerical turbulence simulations. ${ }^{13}$ This will allow us to make detailed observational tests of theoretical magneto hydrodynamic (MHD) models. Recent observations made at South Pole show that the extended submm emission from GMCs is indeed polarized ${ }^{14}$ (Fig. 1). We will use BLAST-pol observations (Fig. 1) to probe magnetic field patterns on intermediate scales. This will allow us to tackle quite detailed questions such as the following 3 examples:

1. Is core morphology determined by large-scale fields? Jones \& Basu ${ }^{15}$ argue that observations support the predominance of oblate cores in molecular clouds, as predicted by magnetically-regulated models. ${ }^{16,17}$ Such models also predict that each core is embedded in a large-scale cloud field running parallel to the core minor axis. Submm polarimetry of quiescent cores $^{12}$ questioned this prediction by finding in SCUBA data that magnetic fields were misaligned, more consistent with turbulent magnetic models. However, SCUBA (and SCUBA-2) cannot trace the fields into lower density regions, and what is really needed are observations tracing core fields out into the surrounding lower-density cloud regions. BLAST-pol will provide these.

2. Does filamentary structure have a magnetic origin? Faint, tenuous ${ }^{12} \mathrm{CO}$ filaments observed in Taurus ${ }^{18,19}$ closely follow the optical polarimetry vectors, indicating a possible magnetic origin. However, denser filaments in Taurus and other clouds show no preferred orientation with respect to the nearby stellar polarimetry vectors. ${ }^{20}$ This may reflect a non-magnetic origin for the denser filaments, or may simply reflect the inadequacies of optical/near-IR polarimetry for tracing fields in shielded regions. ${ }^{10,21}$ It is therefore important to map dense filaments using submm polarimetry to answer the question about the relation between fields and structure.

3. What is the field strength, and how does it vary from cloud to cloud? The Chandrasekhar-Fermi (CF) technique relates dispersion in polarization angle to field strength. ${ }^{22,23}$ For molecular cloud cores, CF field strength estimates have been obtained from submm data, and the results are in rough agreement with Zeeman observations. ${ }^{9}$ Numerical turbulence simulations have been used to calibrate the CF technique for molecular clouds. ${ }^{13,24,25}$ BLAST-pol will determine the power spectrum of the angle dispersion over a wide range of spatial scales and will also measure 3 -color polarization spectra and their spatial variations. ${ }^{26,27}$ These observed characteristics will provide new constraints for the turbulence models that should lead to improved models and better CF calibration. Our observations will probe the dependence of field strength on cloud age, cloud location, and cloud mass.

\subsection{Polarized Galactic Dust Foregrounds}

The search for the primordial gravity waves (GWs) by detecting the B-mode polarization of the Cosmic Microwave Background $(\mathrm{CMB})^{28-30}$ requires a control of instrumental and astrophysical systematics at a level which allows 

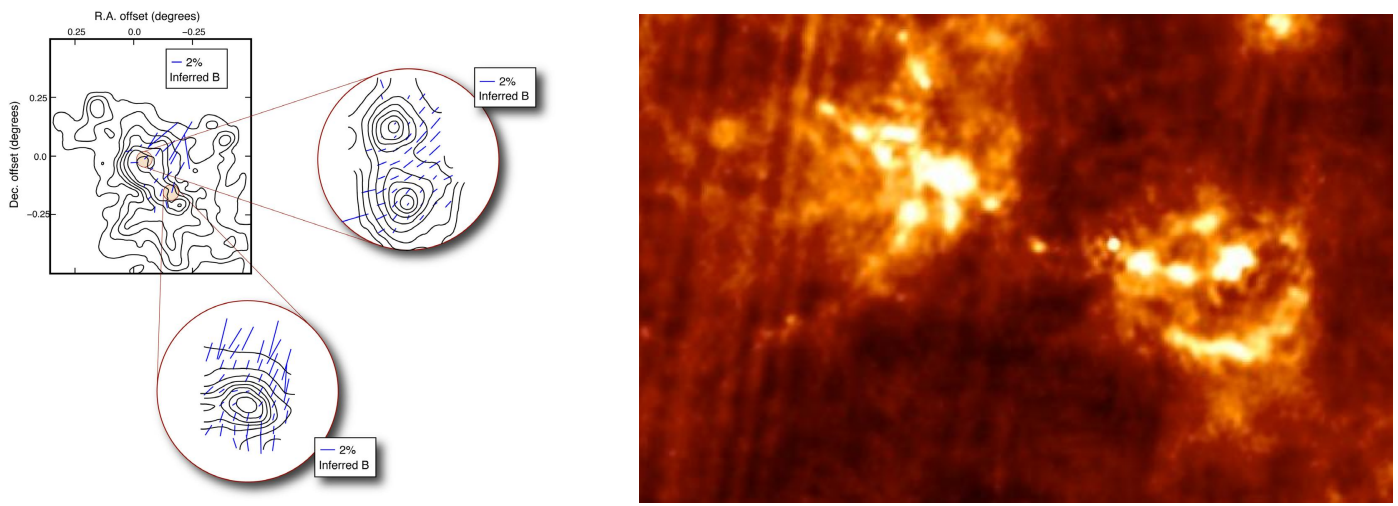

Figure 1. Left panel: submillimeter polarimetry of NGC 6334, a massive GMC at a distance of 1700 pc. The pseudovectors in the map at upper left were obtained using SPARO at South Pole, and the "blowup" at right and bottom are from Hertz/CSO on Mauna Kea. The orientation of each pseudo-vector shows inferred field direction, and the length of each pseudo-vector is proportional to the degree of polarization. With BLAST-pol, we will map scores of such GMCs, obtaining up to 1000 pseudo-vectors per cloud. Right panel: A dust emission map at $250 \mu \mathrm{m}$ of a star forming region in the Galactic plane towards the constellation Cygnus obtained by BLAST during the 2005 flight.

the detection of this faint signal. Astrophysical foregrounds might ultimately be a limiting factor, as the the cosmological B-mode intensity has currently only upper limits and a solid theoretical background is missing to make any realistic prediction. The WMAP polarization observations ${ }^{31}$ clearly demonstrate that polarized galactic foregrounds, even in the range of frequencies where their emission is close to a minimum, are likely to be comparable or larger than the B-mode CMB polarization anisotropy at large angular scales $(\ell<100)$ and intermediate Galactic latitudes. This is a situation unlike the temperature anisotropy where most of the sky is dominated by $\mathrm{CMB}$, and it requires significant foreground emission modeling in order to extract the underlying CMB polarization signal. Most of the polarized galactic foreground that WMAP observed is due to synchrotron emission. At the highest $W M A P$ frequency band $(94 \mathrm{GHz})$, there is evidence for polarized dust emission. This is potentially problematic for the many higher-frequency bolometric CMB polarization experiments (EBEX, Spider, Clover, QUAD, BICEP, SPUD, Planck-HFI, and Polarbear) since the dust is expected to rise significantly in frequency as predicted by models based on the observations at $100 \mu \mathrm{m}$. In addition to WMAP the ARCHEOPS balloon mission measured Galactic dust polarization on scales of $15^{\prime}$ at submm wavelengths. ARCHEOPS results $^{32}$ have shown that radiation from diffuse Galactic dust is polarized (as expected) at a $3-5 \%$ level, but in some regions the polarization is as high as $10 \%$. While the measurements were made near the Galactic plane, they point to the need for more information at higher latitudes, with higher sensitivity, better angular resolution, and at multiple wavelengths. Despite the WMAP and Archeops results, no information exists for any region of the sky at the accuracy required for a B-mode signal detection, and very little information exists at frequencies relevant to CMB science. There are also puzzles in dust polarimetry that must be resolved if we are to make a robust detection of the primordial GWs. For instance, polarimetry of bright molecular cloud cores at $350 \mu \mathrm{m}$ and $1 \mathrm{~mm}$ has revealed the surprising result that the polarized fraction at $1 \mathrm{~mm}$ is larger by a factor of 2 compared to that at $350 \mu \mathrm{m} .{ }^{11}$ The Planck satellite will provide a unique dataset by mapping the sky with polarization sensitive bolometers in bands centered from $850 \mu \mathrm{m}$ to $3 \mathrm{~mm}$. The $850 \mu \mathrm{m}$ band will provide an unparalleled dataset for studying polarized dust. In addition to Planck, balloon-borne and other ground based instruments employ anywhere 2 to 6 frequency bands to assist in discriminating against foreground contamination. But the ability to separate the foreground dust emission to the necessary level to detect B-mode polarization depends from the complexity of the polarized dust emission.

Figure 2 shows the expected polarized dust emission as measured by the $3 \mathrm{yr} W M A P$ data ${ }^{31}$ and the level of foregrounds predicted in a selected $\sim 200 \mathrm{deg}^{2}$ sky region $\left(l=258^{\circ}, b=-46^{\circ}\right)$ accessible by sub-orbital and ground based instruments. Amazingly, the dust signal extrapolated to the CMB frequencies is comparable to the $\mathrm{r}=0.1 \mathrm{~B}$-mode signal level that is being probed by the current generation of experiments. For this reason, it is imperative that we begin to understand high-latitude polarized dust emission. Given these rather small signal 

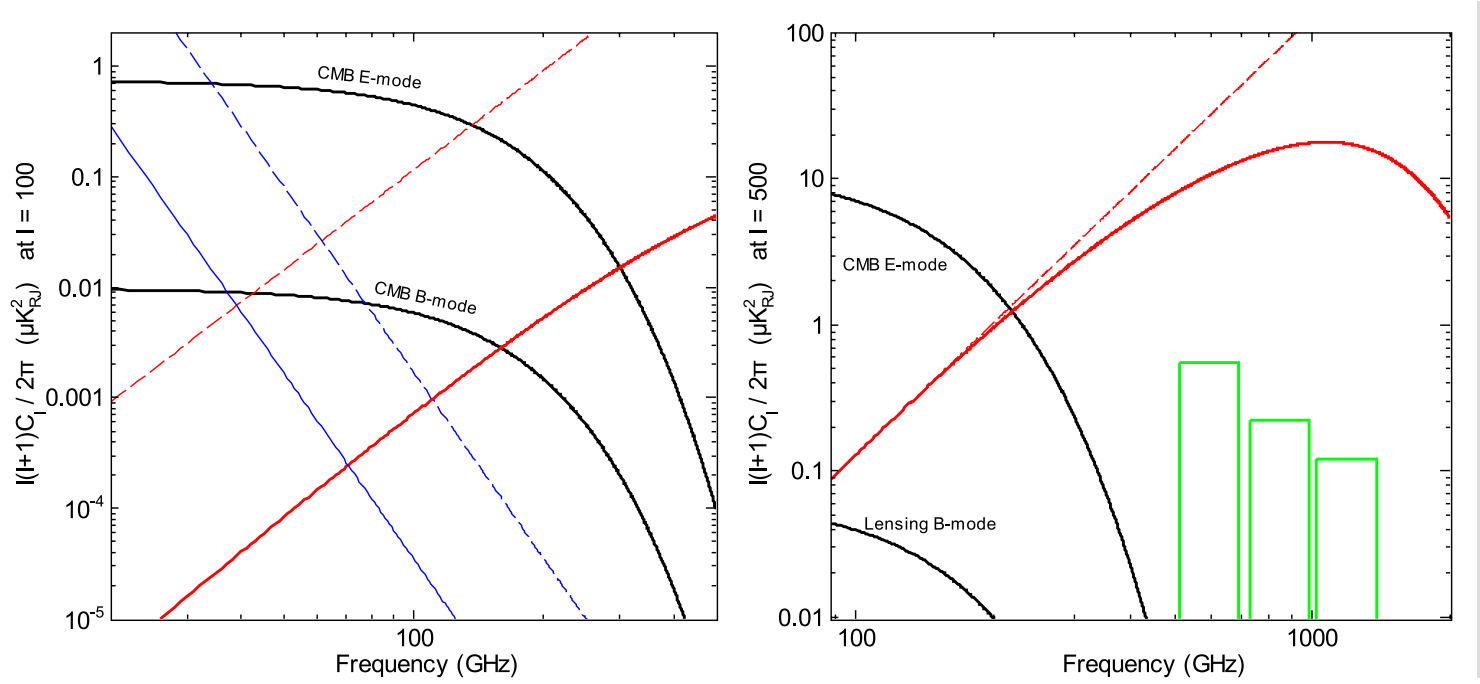

Figure 2. Left panel: The spectrum of B-mode foregrounds from Galactic dust (red) and synchrotron (blue) expected outside WMAP's P06 mask ${ }^{31}$ (dashed) and from a selected sky region (solid), and the E and B-mode CMB (black, solid). All at $\ell=100$ and in antenna temperature. This dust model assumes model 8 from Finkbeiner et al. (1999). ${ }^{33}$ Right panel: A similar plot at $\ell=500$. The green bars show the BLAST-pol noise at a resolution of $8^{\prime}$ from a $48 \mathrm{hr}$ observation of $10 \mathrm{deg}^{2}$ region. At these angular scales, the CMB signal is dominated by the lensing-B. The Galactic dust signal, normalized to WMAP, peaks at the BLAST-pol frequencies. (The CMB models assume a standard $\Lambda$ CDM cosmology with $\mathrm{r}=0.1$.)

levels, BLAST-pol will not be able to conduct large surveys that could be used as templates for lower frequency CMB polarization observations. A 48-hr survey over a $5 \mathrm{deg}^{2}$ sky region (Fig. 2, right panel) will enable BLASTpol to constrain dust properties difficult to measure at lower frequencies. In particular, the determination of dust temperature and the degree of polarization will be highly degenerate as measured by all CMB experiments in the Rayleigh-Jeans tail of the dust spectrum. The ability of BLAST-pol to extract these parameters will be straightforward since the measurements are near the peak of the dust spectrum.

The first of our two maps will be aimed in a very low dust emission region, comparable to $1 \mathrm{MJy} / \mathrm{sr}$ at $100 \mu \mathrm{m}$, that is ideally coincident with a deep CMB polarization measurement. The other will occur in a mid-latitude region with a higher dust brightness that will almost certainly be sampled by Planck. The corresponding higher signal will enable parameter extraction without the aid of lower frequency CMB polarization measurements.

When these measurements are combined, it will enable the characterization of the spatial variation of the polarization percentage, the dust temperature, and the dust emissivity. While the maps will be smoothed to $8^{\prime}$ pixels, we will retain the ability to see any fine-scale, higher-level polarization signals to help the planning of future ground-based, balloon-borne and space experiments.

\section{INSTRUMENT}

\subsection{Optical Design}

The BLAST gondola incorporates a $1.9 \mathrm{~m}$ primary mirror and a $40 \mathrm{~cm}$ diameter correcting secondary mirror giving diffraction-limited performance over a $6.5^{\prime} \times 13^{\prime}$ FOV at the telescope focus at $\lambda=250 \mu \mathrm{m}$. A diagram of the optical system is given in Fig. 3. The estimated antenna efficiency is $>80 \%$, determined by a combination of the rms surface roughness of the primary and the quality of the re-imaging optics.

A carbon fiber spherical primary mirror was used for the Sweden flight, but was damaged during the flight. For the Antarctic flight we used a parabolic aluminum mirror in a Ritchie-Chretién configuration. This mirror, used during the 2003 test flight, was resurfaced to an surface accuracy of $<4 \mu \mathrm{m}$ rms by the Precision Engineering Group at Lawrence Livermore National Laboratory. 


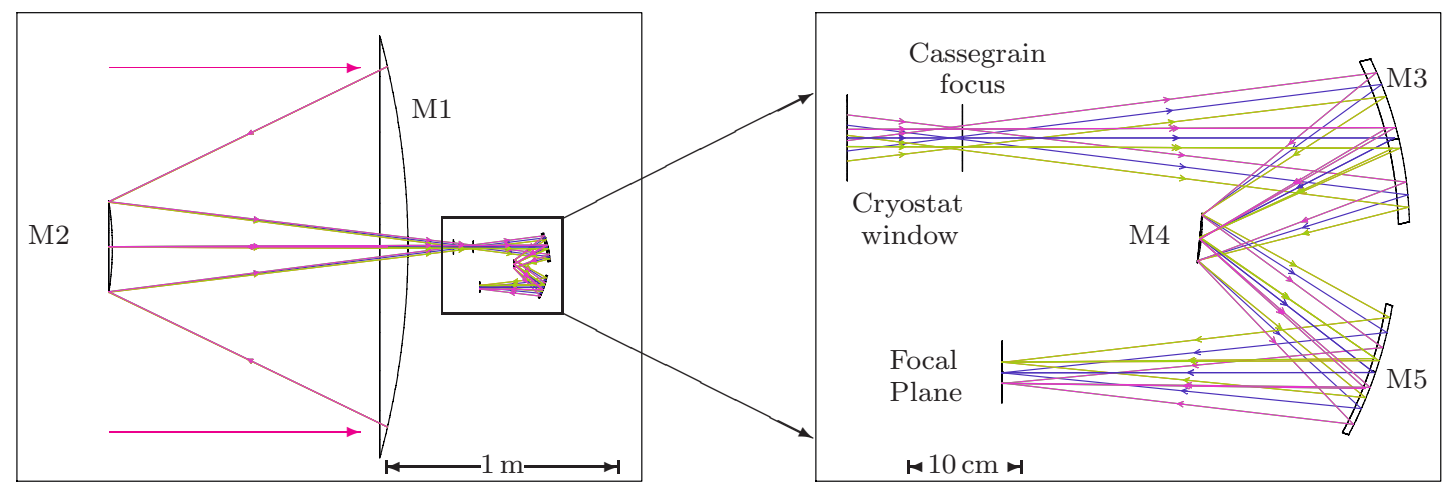

Figure 3. The optical layout of the BLAST05 telescope and receiver is shown on the left and the 1.5K optics, located within the cryostat, are shown in expanded view on the right. The image of the sky formed at the input aperture is re-imaged onto the bolometer detector array at the focal plane. The mirror M4 serves as a Lyot stop defining the illumination of the primary mirror for each element of the bolometer array. The three wavelength bands are separated by a pair of dichroic beamsplitters (not shown) which are arranged in a direction perpendicular to the plane, between M5 and the focal plane.

Temperatures are not constant during the flight and thermal expansion causes variation in the curvature radii of the optical surfaces. The relative distance between the primary and the secondary mirrors has a tolerance of one wavelength before significant image degradation is introduced. Thermal modeling indicated that diurnal temperature fluctuations at balloon altitudes (as high as $10^{\circ} \mathrm{C}$ ) requires a correction in the relative distance between the primary and the secondary mirror of $50 \mu \mathrm{m} /{ }^{\circ} \mathrm{C}$.

To compensate, we move the secondary using three stepper motor actuators to correct for the focus position. These actuators also provide tip/tilt capability for initial alignment.

In the configuration used for the Antarctic campaign, radiation from the telescope is re-imaged onto the focal plane by three spherical mirrors arranged in an Offner-relay configuration and mounted in a $1.5 \mathrm{~K}$ cooled optics box (Figs. 3 and 4). A more complete discussion of the optics for BLAST05 can be found in Olmi (2002). ${ }^{34}$

\subsection{Detectors}

The BLAST focal plane consists of arrays of 149,88 and 43 detectors at 250,350 , and $500 \mu \mathrm{m}$, respectively. The detectors are silicon-nitride micromesh (spider-web) bolometric detectors coupled with $2 \mathrm{f} \lambda$ feedhorn arrays. The entire bolometer/detector array (BDA) is based on the SPIRE instrument detectors. ${ }^{35,36}$

The sensitivity of the detectors is limited by photon shot-noise from the telescope. The total emissivity for the warm optics of $\sim 6 \%$ is dominated by blockage from the secondary mirror and supports. We estimate the optical efficiency of the cold filters and optics to be $\eta_{\mathrm{opt}} \geq 0.3$. The detectors are read out with an AC-biased differential circuit. The data acquisition electronics demodulate the detector signals to provide noise stability to low frequencies $(<30 \mathrm{mHz})$, which allows the sky to be observed in a slowly-scanned mode. Slow scanning is preferable to a mechanical chopper for mapping large regions of the sky to the confusion limit. The data are collected using a high-speed, flexible, 22-bit data acquisition system developed at the University of Toronto. The system can synchronously sample up to 600 channels at any rate up to $4 \mathrm{kHz}$. Each channel consists of a buffered input and a sigma-delta analog to digital converter. The output from 24 channels are then processed by an Altera programmable logic device (PLD) which digitally anti-alias filters and demodulates each input. The results are stored to disk.

\subsection{Polarimetry}

Adding polarimetry to the BLAST focal plane is relatively straightforward compared to the technical challenges that have been addressed in building the whole instrument. The design incorporates an Achromatic Half Wave Plate (AHWP) located inside the optics box as indicated in Fig. 4. It will be mounted inside the optics box, far from the Cassegrain focus to minimize modulation of imperfections, while allowing it to operate and dissipate 


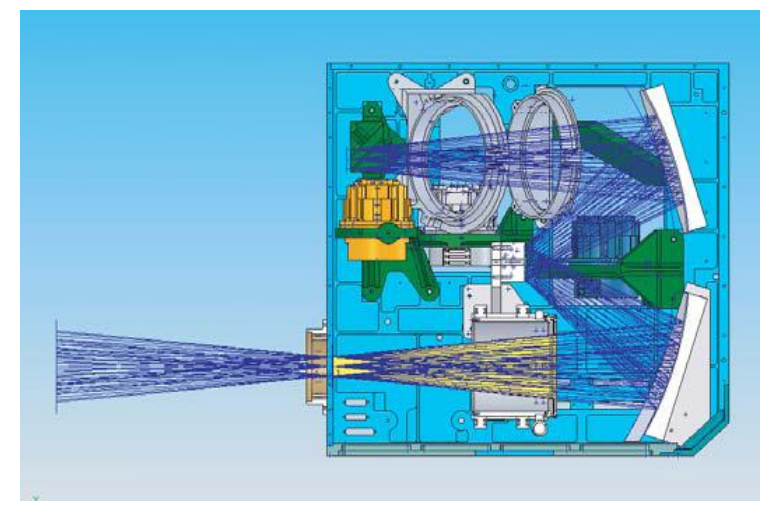

Figure 4. A cutaway view of the BLAST optics box. The light enters from the lower left and is reimaged onto the arrays. Dichroic filters split the beam into each of the arrays. For BLAST-pol, a modulating cold half-wave plate will be placed inside the optics box and polarizing grids will be mounted in front of each of the BDAs. The motorized mechanism for rotating the waveplate will be located outside the optics box.

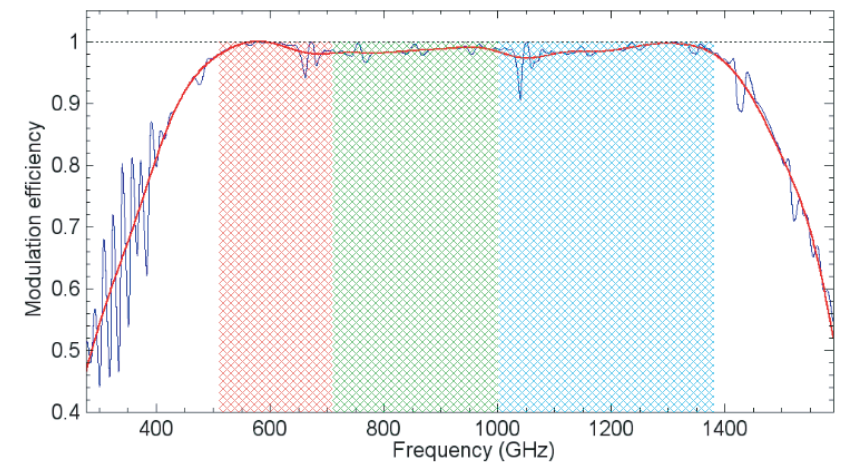

Figure 5. The modeled performance of the antireflection coated 5-plate design of the BLAST half-wave plate. The diameter will be $9 \mathrm{~cm}$. A similar design has already been built and tested for SCUBA-2 (20 cm diameter). The optical efficiency ranges between 85 and $96 \%$ depending on the type of anti-reflection coating. We will build several types and use the one with the best results.

heat to the $4 \mathrm{~K}$ stage. The 5 layer AHWP will have an optical efficiency between 85 and $96 \%$ depending upon the anti-reflection coating and material used. Options currently under investigation include quartz and sapphire. Each of these presents different challenges and we will chose the solution which maximizes the ratio between optical performances to technical challenge. The predicted modulation efficiency across the BLAST bands is given in Fig. 5. The waveplate will be operated in either a stepped mode or continuously rotated. The stepped mode solution implements a similar mechanism to the one used by the SPARO instrument. ${ }^{37}$ The continuous rotation will be obtained using magnetic levitating bearings similar to the mechanism developed for Clover. The rotation rate will range from 1 to $5 \mathrm{~Hz}$ resulting in a polarization modulation of 4 to $20 \mathrm{~Hz}$. The two mechanisms, driven by a $300 \mathrm{~K}$ external motor, are interchangeable in order to allow maximum flexibility. The solution and rotation frequency which will be adopted for the flight will depend from the response of the instrument to the different modulation methodologies.

A polarizing photolithographed grid in front of each feed-horn array will be patterned to alternate the polarization angle from horn-to-horn along the scan direction. BLAST has the demonstrated pointing stability necessary to control the scan direction and pitch to have a point on the sky pass from horn-to-horn along a row of the array. The alternating grid will modulate the polarization at beam-crossing timescales even if the stepping system for the half-wave plate fails. The placement of the grid is similar to the method used by Boomerang. ${ }^{38}$ 


\subsection{Cryogenics}

The receiver consists of an optical cavity inside a long hold-time liquid-nitrogen and liquid-helium cryostat. Both the nitrogen and helium are maintained at slightly more than atmospheric pressure during the flight to minimize loss due to pressure drop at altitude. A ${ }^{3} \mathrm{He}$ refrigerator maintains the detectors at $300 \mathrm{mK}$ during flight. The self-contained, recycling refrigerator can maintain a base temperature of $280 \mathrm{mK}$ with $30 \mu \mathrm{W}$ of cooling power for 4 days. It can be recycled within $2 \mathrm{hr}$. The ${ }^{3} \mathrm{He}$ refrigerator uses a pumped ${ }^{4} \mathrm{He}$ pot at $\sim 1 \mathrm{~K}$ for cycling and to increase the hold time of the system. The pumped pot maintains $1 \mathrm{~K}$ with $20 \mathrm{~mW}$ of cooling power with outside pressure 15 Torr or less. The entire optics box containing the re-imaging optics is also cooled to $1 \mathrm{~K}$.

\subsection{Gondola}

The BLAST gondola provides a pointed platform for the telescope and the attachment point to the balloon flight train. The instrument is mounted on an alt-azimuth aluminum frame (the gondola) made of an outer frame $(\mathrm{OF})$ providing azimuth directionality and holding an inner frame for elevation pointing. The OF is hung from the $1.1 \times 10^{6} \mathrm{~m}^{3}$ helium balloon, provided by NASA's Columbia Scientific Balloon Facility, through a steel cable and parachute. Control computers and telemetric systems are mounted on the OF. Data are stored on these computers and partially transmitted through satellite links to the ground station. Sun shields, made of aluminized mylar panels, keep sunlight from heating the telescope, and are mounted on the OF. The IF is built to house the mirror, the receiver, its readout electronics and the pointing sensors, all rigidly mounted with respect to each other to ensure that mechanical alignment is maintained throughout the flight.

The telescope is controlled in azimuth by a $1.5 \mathrm{~m}$ reaction wheel, made of $7.6 \mathrm{~cm}$ thick aluminum honeycomb, and $480.9 \mathrm{~kg}$ brass disks mounted around the perimeter, to maximize the ratio of moment of inertia to mass. The reaction wheel is mounted at the center of the outer frame with its rotation axis going through the pivot. Torquing the gondola against the reaction wheel controls azimuth pointing. The elevation of the inner frame is controlled by a motor mounted on one side of the inner frame at the attachment point to the outer frame. A free bearing provides the connection point on the other side.

Pointing is measured in-flight to an accuracy of $\sim 30^{\prime \prime}$ rms by a variety of fine and coarse sensors, including fiber optic gyroscopes, optical star cameras, a differential GPS, magnetometer and Sun sensor. Post-flight pointing reconstruction uses only the gyroscopes and day-time star trackers. ${ }^{39}$ The algorithm is based on a similar multiplicative extended Kalman filter ${ }^{40}$ technique used by $W M A P,{ }^{41}$ and modified $^{42}$ to allow the evaluation of the star trackers and gyroscopes alignment parameters. The offset between the star cameras and the submm telescope was measured by repeated observations of pointing calibrators throughout the flight. We find that the relative pointing between the star cameras and submm telescope varies as a function of IF elevation. We apply an elevation-dependent correction to pitch and yaw with scales or $\sim 125^{\prime \prime}$ and $\sim 20^{\prime \prime}$, respectively. Post flight pointing accuracy is verified by a stacking analysis on one of the extragalactic maps. Using the deep radio ECDF-S VLA survey at $1.4 \mathrm{GHz},{ }^{43}$ we stack patches of the BLAST maps centered at the radio source coordinates, simply summing the flux pixel by pixel (see Fig. 6). We find that the peak in the stacked map is located within $2^{\prime \prime}$ from the nominal position of the catalogue, indicating that the absolute pointing accuracy is at least 15 times smaller than the beam size. Moreover, assuming random Gaussian pointing errors, we superimpose the synthetic scaled Point Spread Function (PSF) to the stacked map and convolve it with a Gaussian profile, modeling the broadening of the PSF due to pointing jitter. By varying the jitter width, we compute the $\chi^{2}$ of the convolved PSF over the stacked data. In this way we estimate the upper limit in potential random pointing errors to be $3^{\prime \prime}$.

\section{ACKNOWLEDGMENTS}

The BLAST collaboration acknowledges the support of NASA through grant numbers NAG5-12785, NAG513301 and NNGO-6GI11G, the Canadian Space Agency (CSA), the Science and Technology Facilities Council (STFC), Canada's Natural Sciences and Engineering Research Council (NSERC), the Canada Foundation for Innovation, the Ontario Innovation Trust, the Puerto Rico Space Grant Consortium, the Fondo Istitucional para la Investigacion of the University of Puerto Rico, and the National Science Foundation Office of Polar Programs; C. B. Netterfield also acknowledges support from the Canadian Institute for Advanced Research. L. Olmi would like to acknowledge Pietro Bolli for his help with Physical Optics simulations during the testing 


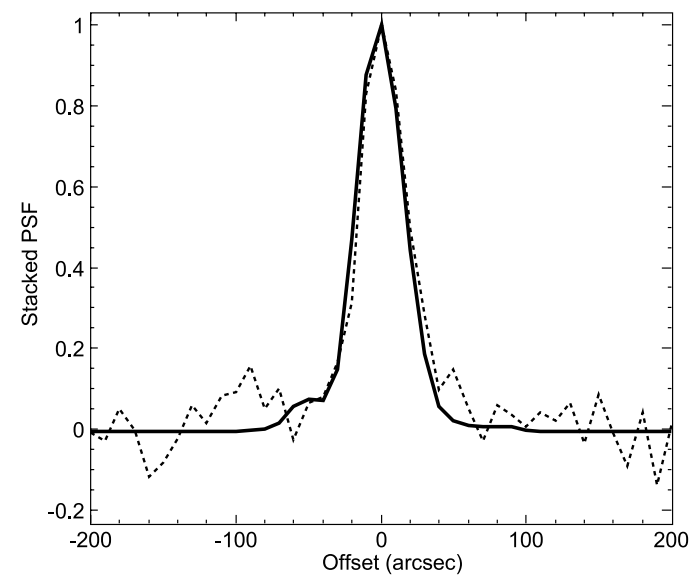

Figure 6. A cut through the stacked BLAST $250 \mu \mathrm{m}$ flux at the positions of VLA $1.4 \mathrm{GHz}$ radio sources ${ }^{43}$ (dashed line) and through the $250 \mu \mathrm{m}$ point spread function (solid line). We see that the stack is very well described by the PSF, in both position and width. We conclude that our absolute pointing is good to $<2^{\prime \prime}$ and that random pointing errors are $<3^{\prime \prime}$ rms.

phase of BLAST06. We would also like to thank the Columbia Scientific Balloon Facility (CSBF) staff for their outstanding work, the Precision Machining Group at Lawrence Livermore Laboratory, the support received from Empire Dynamic Structures in the design and construction of the gondola, Daniele Mortari for helpful discussions in the development of the Pyramid code, Dan Swetz for buliding the Fourier transform spectrometer, and Luke Bruneaux, Kyle Lepage, Danica Marsden, Vjera Miovic, and James Watt for their contribution to the project.

\section{REFERENCES}

[1] Griffin, M. J., Swinyard, B. M., and Vigroux, L. G., "SPIRE - Herschel's Submillimetre Camera and Spectrometer," in [IR Space Telescopes and Instruments. Edited by John C. Mather. Proceedings of the SPIE], 4850, 686-697 (Mar. 2003).

[2] Devlin, M. J., Ade, P. A. R., Aretxaga, I., Bock, J. J., Chung, J., Chapin, E., Dicker, S. R., Griffin, M., Gundersen, J., Halpern, M., Hargrave, P., Hughes, D., Klein, J., Marsden, G., Martin, P., Mauskopf, P. D., Netterfield, B., Olmi, L., Pascale, E., Rex, M., Scott, D., Semisch, C., Truch, M., Tucker, C., Tucker, G., Turner, A. D., and Weibe, D., "The Balloon-borne Large Aperture Submillimeter Telescope (BLAST)," in [Astronomical Structures and Mechanisms Technology. Edited by Antebi, Joseph; Lemke, Dietrich. Proceedings of the SPIE, Volume 5498, pp. 42-54 (2004).], $42-54$ (Oct. 2004).

[3] Hughes, D. H., Aretxaga, I., Chapin, E. L., Gaztañaga, E., Dunlop, J. S., Devlin, M. J., Halpern, M., Gundersen, J., Klein, J., Netterfield, C. B., Olmi, L., Scott, D., and Tucker, G., "Breaking the "redshift deadlock'- I. Constraining the star formation history of galaxies with submillimetre photometric redshifts," MNRAS 335, 871-882 (Oct. 2002).

[4] Truch, M. D. P.and Ade, P. A. R., Bock, J. J., Chapin, E. L., Chung, J., Devlin, M. J., Dicker, S., Griffin, M., Gundersen, J. O., Halpern, M., Hargrave, P. C., Hughes, D. H., Klein, J., MacTavish, C. J., Marsden, G., Martin, P. G., Martin, T. G., Mauskopf, P., Netterfield, C. B., Olmi, L., Pascale, E., Patanchon, G., Rex, M., Scott, D., Semisch, C., Thomas, N., Tucker, C., Tucker, G. S., Viero, M. P., and Wiebe, D. V., "The Balloon-Borne Large Aperture Submillimeter Telescope (BLAST) 2005: Calibration and Targeted Sources," ApJ accepted for publication (Mar. 2008).

[5] Pascale, E., Ade, P. A. R., Bock, J. J., Chapin, E. L., Chung, J., Devlin, M. J., Dicker, S., Griffin, M., Gundersen, J. O., Halpern, M., Hargrave, P. C., Hughes, D. H., Klein, J., MacTavish, C. J., Marsden, G., Martin, P. G., Martin, T. G., Mauskopf, P., Netterfield, C. B., Olmi, L., Patanchon, G., Rex, M., Scott, D., Semisch, C., Thomas, N., Truch, M. D. P., Tucker, C., Tucker, G. S., Viero, M. P., and Wiebe, D. V., "The 
Balloon-borne Large Aperture Submillimeter Telescope: BLAST," ApJ accepted for publication (Mar. 2008).

[6] Patanchon, G., Ade, P. A. R., Bock, J. J., Chapin, E. L., Devlin, M. J., Dicker, S., Griffin, M., Gundersen, J. O., Halpern, M., Hargrave, P. C., Hughes, D. H., Klein, J., Marsden, G., Martin, P. G., Mauskopf, P., Netterfield, C. B., Olmi, L., Pascale, E., Rex, M., Scott, D., Semisch, C., Truch, M. D. P., Tucker, C., Tucker, G. S., Viero, M. P., and Wiebe, D. V., "SANEPIC: A Map-Making Method for Timestream Data From Large Arrays," ApJ accepted for publication (Nov. 2007).

[7] Chapin, E. L., Ade, P. A. R., Bock, J. J., Brunt, C., Devlin, M. J., Dicker, S., Griffin, M., Gundersen, J. O., Halpern, M., Hargrave, P. C., Hughes, D. H., Klein, J., Marsden, G., Martin, P. G., Mauskopf, P., Netterfield, C. B., Olmi, L., Pascale, E., Patanchon, G., Rex, M., Scott, D., Semisch, C., Truch, M. D. P., Tucker, C., Tucker, G. S., Viero, M. P., and Wiebe, D. V., "The Balloon-borne Large Aperture Submillimeter Telescope (BLAST) 2005: A 4 sq. deg Galactic Plane Survey in Vulpecula (l=59)," ApJ accepted for publication (Oct. 2007).

[8] McKee, C. F. and Ostriker, E. C., "Theory of Star Formation," ARA $8 A$ 45, 565-687 (Sept. 2007).

[9] Crutcher, R. M., Nutter, D. J., Ward-Thompson, D., and Kirk, J. M., "SCUBA Polarization Measurements of the Magnetic Field Strengths in the L183, L1544, and L43 Prestellar Cores," ApJ 600, 279-285 (Jan. 2004).

[10] Whittet, D. C. B., Hough, J. H., Lazarian, A., and Hoang, T., "The Efficiency of Grain Alignment in Dense Interstellar Clouds: a Reassessment of Constraints from Near-Infrared Polarization," ApJ 674, 304-315 (Feb. 2008).

[11] Hildebrand, R. H., Davidson, J. A., Dotson, J. L., Dowell, C. D., Novak, G., and Vaillancourt, J. E., "A Primer on Far-Infrared Polarimetry," PASP 112, 1215-1235 (Sept. 2000).

[12] Ward-Thompson, D., Kirk, J. M., Crutcher, R. M., Greaves, J. S., Holland, W. S., and André, P., "First Observations of the Magnetic Field Geometry in Prestellar Cores," ApJ 537, L135-L138 (July 2000).

[13] Ostriker, E. C., Stone, J. M., and Gammie, C. F., "Density, Velocity, and Magnetic Field Structure in Turbulent Molecular Cloud Models," ApJ 546, 980-1005 (Jan. 2001).

[14] Li, H., Griffin, G. S., Krejny, M., Novak, G., Loewenstein, R. F., Newcomb, M. G., Calisse, P. G., and Chuss, D. T., "Results of SPARO 2003: Mapping Magnetic Fields in Giant Molecular Clouds," ApJ 648, 340-354 (Sept. 2006).

[15] Jones, C. E. and Basu, S., "The Intrinsic Shapes of Molecular Cloud Fragments over a Range of Length Scales," ApJ 569, 280-287 (Apr. 2002).

[16] Mouschovias, T. C. and Ciolek, G. E., "Magnetic Fields and Star Formation: A Theory Reaching Adulthood," in [NATO ASIC Proc. 540: The Origin of Stars and Planetary Systems], Lada, C. J. and Kylafis, N. D., eds., 305-+ (1999).

[17] Allen, A., Li, Z.-Y., and Shu, F. H., "Collapse of Magnetized Singular Isothermal Toroids. II. Rotation and Magnetic Braking," ApJ 599, 363-379 (Dec. 2003).

[18] Heyer, M., Gong, H., Ostriker, E., and Brunt, C., "Magnetically Aligned Velocity Anisotropy in the Taurus Molecular Cloud," ArXiv e-prints 802 (Feb. 2008).

[19] Goldsmith, P. F., Heyer, M., Narayanan, G., Snell, R., Li, D., and Brunt, C., "Large-Scale Structure of the Molecular Gas in Taurus Revealed by High Linear Dynamic Range Spectral Line Mapping," ArXiv e-prints 802 (Feb. 2008).

[20] Goodman, A. A., Bastien, P., Menard, F., and Myers, P. C., "Optical polarization maps of star-forming regions in Perseus, Taurus, and Ophiuchus," ApJ 359, 363-377 (Aug. 1990).

[21] Cho, J. and Lazarian, A., "Grain Alignment by Radiation in Dark Clouds and Cores," ApJ 631, 361-370 (Sept. 2005).

[22] Chandrasekhar, S. and Fermi, E., "Magnetic Fields in Spiral Arm," ApJ 118, 113 (July 1953).

[23] Zweibel, E. G., "Magnetic field-line tangling and polarization measurements in clumpy molecular gas," ApJ 362, 545-550 (Oct. 1990).

[24] Pelkonen, V.-M., Juvela, M., and Padoan, P., "Simulations of polarized dust emission," A $\& A$ 461, 551-564 (Jan. 2007). 
[25] Falceta-Goncalves, D., Lazarian, A., and Kowal, G., "Studies of regular and random magnetic fields in the ISM: statistics of polarization vectors and the Chandrasekhar-Fermi technique," ArXiv e-prints $\mathbf{8 0 1}$ (Jan. 2008).

[26] Bethell, T. J., Chepurnov, A., Lazarian, A., and Kim, J., "Polarization of Dust Emission in Clumpy Molecular Clouds and Cores," ApJ 663, 1055-1068 (July 2007).

[27] Vaillancourt, J. E., Dowell, C. D., Hildebrand, R. H., Kirby, L., Krejny, M. M., Li, H.-b., Novak, G., Houde, M., Shinnaga, H., and Attard, M., "New Results on the Submillimeter Polarization Spectrum of the Orion Molecular Cloud," ApJ 679, L25-L28 (May 2008).

[28] Hu, W. and White, M., "A CMB polarization primer," New Astronomy 2, 323-344 (Oct. 1997).

[29] Kamionkowski, M., Kosowsky, A., and Stebbins, A., "Statistics of cosmic microwave background polarization," Phys. Rev. D 55, 7368-7388 (Jun 1997).

[30] Zaldarriaga, M. and Seljak, U. c. v., "All-sky analysis of polarization in the microwave background," Phys. Rev. D 55, 1830-1840 (Feb 1997).

[31] Page, L., Hinshaw, G., Komatsu, E., Nolta, M. R., Spergel, D. N., Bennett, C. L., Barnes, C., Bean, R., Doré, O., Dunkley, J., Halpern, M., Hill, R. S., Jarosik, N., Kogut, A., Limon, M., Meyer, S. S., Odegard, N., Peiris, H. V., Tucker, G. S., Verde, L., Weiland, J. L., Wollack, E., and Wright, E. L., "Three-Year Wilkinson Microwave Anisotropy Probe (WMAP) Observations: Polarization Analysis," ApJS 170, 335376 (June 2007).

[32] Benoît, A., Ade, P., Amblard, A., Ansari, R., Aubourg, É., Bargot, S., Bartlett, J. G., Bernard, J.-P., Bhatia, R. S., Blanchard, A., Bock, J. J., Boscaleri, A., Bouchet, F. R., Bourrachot, A., Camus, P., Couchot, F., de Bernardis, P., Delabrouille, J., Désert, F.-X., Doré, O., Douspis, M., Dumoulin, L., Dupac, X., Filliatre, P., Fosalba, P., Ganga, K., Gannaway, F., Gautier, B., Giard, M., Giraud-Héraud, Y., Gispert, R., Guglielmi, L., Hamilton, J.-C., Hanany, S., Henrot-Versillé, S., Kaplan, J., Lagache, G., Lamarre, J.-M., Lange, A. E., Macías-Pérez, J. F., Madet, K., Maffei, B., Magneville, C., Marrone, D. P., Masi, S., Mayet, F., Murphy, A., Naraghi, F., Nati, F., Patanchon, G., Perrin, G., Piat, M., Ponthieu, N., Prunet, S., Puget, J.-L., Renault, C., Rosset, C., Santos, D., Starobinsky, A., Strukov, I., Sudiwala, R. V., Teyssier, R., Tristram, M., Tucker, C., Vanel, J.-C., Vibert, D., Wakui, E., and Yvon, D., "First detection of polarization of the submillimetre diffuse galactic dust emission by Archeops," A $\& A$ 424, 571-582 (Sept. 2004).

[33] Finkbeiner, D. P., Davis, M., and Schlegel, D. J., "Extrapolation of Galactic Dust Emission at 100 Microns to Cosmic Microwave Background Radiation Frequencies Using FIRAS," ApJ 524, 867-886 (Oct. 1999).

[34] Olmi, L., "Optical designs for submillimeter-wave spherical-primary (sub)orbital telescopes and novel optimization techniques," in [Highly Innovative Space Telescope Concepts Edited by Howard A. MacEwen. Proceedings of the SPIE], 4849, 245-256 (Dec. 2002).

[35] Glenn, J., Bock, J. J., Chattopadhyay, G., Edgington, S. F., Lange, A. E., Zmuidzinas, J., Mauskopf, P. D., Rownd, B., Yuen, L., and Ade, P. A., "Bolocam: a millimeter-wave bolometric camera," in [Proc. SPIE, Advanced Technology MMW, Radio, and Terahertz Telescopes, Thomas G. Phillips; Ed.], 3357, 326-334 (July 1998).

[36] Rownd, B., Bock, J. J., Chattopadhyay, G., Glenn, J., and Griffin, M. J., "Design and performance of feedhorn-coupled bolometer arrays for SPIRE," in [Millimeter and Submillimeter Detectors for Astronomy. Edited by Phillips, Thomas G.; Zmuidzinas, Jonas. Proceedings of the SPIE], 4855, 510-519 (Feb. 2003).

[37] Renbarger, T., Chuss, D. T., Dotson, J. L., Griffin, G. S., Hanna, J. L., Loewenstein, R. F., Malhotra, P. S., Marshall, J. L., Novak, G., and Pernic, R. J., "Early Results from SPARO: Instrument Characterization and Polarimetry of NGC 6334," PASP 116, 415-424 (May 2004).

[38] Masi, S., Ade, P. A. R., Bock, J. J., Bond, J. R., Borrill, J., Boscaleri, A., Cabella, P., Contaldi, C. R., Crill, B. P., de Bernardis, P., de Gasperis, G., de Oliveira-Costa, A., de Troia, G., di Stefano, G., Ehlers, P., Hivon, E., Hristov, V., Iacoangeli, A., Jaffe, A. H., Jones, W. C., Kisner, T. S., Lange, A. E., MacTavish, C. J., Marini Bettolo, C., Mason, P., Mauskopf, P. D., Montroy, T. E., Nati, F., Nati, L., Natoli, P., Netterfield, C. B., Pascale, E., Piacentini, F., Pogosyan, D., Polenta, G., Prunet, S., Ricciardi, S., Romeo, G., Ruhl, J. E., Santini, P., Tegmark, M., Torbet, E., Veneziani, M., and Vittorio, N., "Instrument, method, brightness, and polarization maps from the 2003 flight of BOOMERanG," AËA 458, 687-716 (Nov. 2006). 
[39] Rex, M., Chapin, E., Devlin, M. J., Gundersen, J., Klein, J., Pascale, E., and Wiebe, D., "BLAST autonomous daytime star cameras," in [Ground-based and Airborne Instrumentation for Astronomy. Edited by McLean, Ian S.; Iye, Masanori. Proceedings of the SPIE, Volume 6269, pp. 62693H (2006).], Presented at the Society of Photo-Optical Instrumentation Engineers (SPIE) Conference 6269 (July 2006).

[40] Markley, F. L., "Attitude Error Representations for Kalman Filtering," Journal of Guidance, Control, and Dynamics 26, 311-317 (2003).

[41] Harman, R. R., "Wilkinson Microwave Anisotropy Probe (WMAP) Attitude Estimation Filter Comparison," tech. rep., Flight Mechanics Symposium; Greenbelt, MD (2005).

[42] Pittelkau, M. E., "Kalman Filtering for Spacecraft System Alignment Calibration," Journal of Guidance, Control, and Dynamics 24, 1187-1195 (2001).

[43] Miller, N. A., Fomalont, E. B., Kellermann, K. I., Mainieri, V., Norman, C., Padovani, P., Rosati, P., and Tozzi, P., "The VLA 1.4GHz Survey of the Extended Chandra Deep Field South: First Data Release," ArXiv e-prints $\mathbf{8 0 4}$ (Apr. 2008). 